\title{
Ectopic gut colonization: a metagenomic study of the oral and gut microbiome in Crohn's disease
}

\author{
Shijia Hu ${ }^{*}{ }^{*}$, Eileen Png ${ }^{2}$, Michelle Gowans ${ }^{3}$, David E. H. Ong ${ }^{3}$, Paola Florez de Sessions ${ }^{2}$, Jie Song ${ }^{2}$ \\ and Niranjan Nagarajan ${ }^{2,4}$
}

\begin{abstract}
Background: This study aims to characterize, the gut and oral microbiome in Asian subjects with Crohn's disease (CD) using whole genome shotgun sequencing, thereby allowing for strain-level comparison.

Methods: A case-control study with age, sex and ethnicity matched healthy controls was conducted. CD subjects were limited to well-controlled patients without oral manifestations. Fecal and saliva samples were collected for characterization of gut and oral microbiome respectively. Microbial DNA were extracted, libraries prepared and sequenced reads profiled. Taxonomic diversity, taxonomic association, strain typing and microbial gene pathway analyses were conducted.

Results: The study recruited 25 subjects with CD and 25 healthy controls. The oral microbe Streptococcus salivarius was found to be enriched and of concordant strains in the gut and oral microbiome of Crohn's disease subjects. This was more likely in CD subjects with higher Crohn's Disease Activity Index (184.3 \pm 2.9 vs $67.1 \pm 82.5, p=0.012)$ and active disease status (Diarrhoea/abdominal pain/blood-in-stool/fever and fatigue) $(p=0.016)$. Gut species found to be significantly depleted in CD compared to control (Relative abundance: Median[Range]) include: Faecalibacterium prausnitzii (0.03[0.00-4.56] vs 13.69[5.32-18.71], $p=0.010)$, Roseburia inulinivorans $(0.00[0.00-0.03]$ vs 0.21 [0.01-0.53], $\mathrm{p}=0.010)$ and Alistipes senegalensis $(0.00[0.00-0.00]$ vs $0.00[0.00-0.02], \mathrm{p}=0.029)$. While Clostridium nexile $(0.00[0.00-$ 0.12 ] vs $0.00[0.00-0.00], p=0.038$ ) and Ruminococcus gnavus (0.43[0.02-0.33] vs $0.00[0.00-0.13], p=0.043$ ) were found to be enriched. C. nexile enrichment was not found in CD subjects of European descent. Microbial arginine (Linear-discriminant-analysis: $3.162, p=0.001$ ) and isoprene (Linear-discriminant-analysis: $3.058, p<0.001$ ) pathways were found at a higher relative abundance level in gut microbiome of Crohn's disease.
\end{abstract}

Conclusions: There was evidence of ectopic gut colonization by oral bacteria, especially during the active phase of $C D$. Previously studied gut microbial differences were detected, in addition to novel associations which could have resulted from geographical/ethnic differences to subjects of European descent. Differences in microbial pathways provide possible targets for microbiome modification.

Keywords: Crohn's disease, Gastrointestinal microbiome, Oral microbiome, Metagenomics

*Correspondence: Shijia_hu@nuhs.edu.sg

${ }^{1}$ Discipline of Orthodontics and Paediatric Dentistry, Faculty

of Dentistry, National University of Singapore, 9 Lower Kent Ridge Road, Singapore 119085, Singapore

Full list of author information is available at the end of the article

\section{Background}

Inflammatory bowel diseases (IBD) are a group of digestive tract disease that affect millions of people worldwide. There are signs that incidence rates of IBD are increasing and presenting earlier in life [1]. IBD is divided into 2 main disease processes: Crohn's Disease $(C D)$, which

(c) The Author(s) 2021. This article is licensed under a Creative Commons Attribution 4.0 International License, which permits use, sharing, adaptation, distribution and reproduction in any medium or format, as long as you give appropriate credit to the original author(s) and the source, provide a link to the Creative Commons licence, and indicate if changes were made. The images or other third party material in this article are included in the article's Creative Commons licence, unless indicated otherwise in a credit line to the material. If material is not included in the article's Creative Commons licence and your intended use is not permitted by statutory regulation or exceeds the permitted use, you will need to obtain permission directly from the copyright holder. To view a copy of this licence, visit http://creativeco mmons.org/licenses/by/4.0/. The Creative Commons Public Domain Dedication waiver (http://creativecommons.org/publicdomain/ zero/1.0/) applies to the data made available in this article, unless otherwise stated in a credit line to the data. 
may affect any segment of the gastro-intestinal tract, and Ulcerative Colitis (UC), which is limited to the large intestine [2]. Patients with CD frequently present with severe abdominal pain, fever, and clinical signs of bowel obstruction or diarrhea [3]. Inflammation modulation is the mainstay of medical management and ranges from the use of anti-inflammatories, to corticosteroids and immunomodulators [4]. In severe cases, surgery may be required. Unfortunately, the need for surgery has not decreased despite advancements in diagnostic and treatment protocols [5].

Despite theorizing that $\mathrm{CD}$ arises from an impaired interaction between commensal microbiome and the human host, the distinction between primary driver events and secondary occurrences remains murky. However, the recent focus on gut microbiome dysbiosis in $\mathrm{CD}$ has led to the discovery of new diagnostic and therapeutic directions [6]. Murine models have shown that the disease only manifests in susceptible genotypes and is driven by microbial dysbiosis [7], while human studies have found a general decrease in alpha diversity with clade-specific changes in $C D$ patients such as increased Enterobacteriaceae and decreased Firmicutes [8, 9].

One of the limitations of previous microbiome studies has been the sequencing strategy. Most of the studies employed 16S rRNA sequencing, which limits their findings to bacteria at the genus level. This gap can be addressed with whole genome shotgun (WGS) sequencing, which is able to detect the presence of microbes with better accuracy and provide data at species level [10]. Additionally, most of these studies were in western populations and with subjects of European descent. This limited the scope of previous results as ethnicity, eating habits and living environment are variables that affect the gut microbiome [11].

Previous work on the oral-gut axis has demonstrated a connection between oral inflammation and its contribution to gut inflammation in animal models. Oral pathobiont-reactive inflammatory cells arising from oral inflammation were found to migrate to the gut, promoting and contributing to colitis [12]. The effect of the oral microbiome on $\mathrm{CD}$ is by comparison relatively understudied; however, its impact cannot be ignored [13]. A recent study found that oral Klebsiella can colonize the gut and result in severe gut inflammation in susceptible individuals, thereby exacerbating inflammatory disease [14]. Another study found increased presence of species found abundantly in oral communities in the gut microbiomes of $\mathrm{CD}$ subjects having diarrhea, suggesting that the oral cavity may serve as a reservoir for opportunistic gut pathogens [15].

Previous studies examining the oral microbiome were also obfuscated by having different sampling sites, such as sampling from the tongue [16], plaque [17] and saliva $[18,19]$. Of these sampling sites, the salivary microbiome appears to offer the most diagnostic value without the excessive influence of local factors [20]. These studies found enrichment of Veillonellaceae and depletion of Haemophilus in CD subjects, highlighting the diagnostic value of the oral microbiome $[18,19]$. Moreover, as a diagnostic tool, saliva samples are non-invasive unlike colonoscopy, and easy to collect at any time unlike fecal samples.

This study aims to characterize, for the first time, the oral and gut microbiome in Asian subjects with $C D$ using whole genome shotgun technique. A case-control analysis was conducted with matched healthy controls to investigate the presence of altered community structure and different community ecotypes in the oral and gut microbiomes of a mixed Asian population consisting of Han Chinese, Malay and Indian.

\section{Results}

The study recruited 25 subjects with CD and 25 healthy controls who were age, sex and ethnicity matched. The subjects' demographics, clinical and oral conditions are summarized in Table 1. Most of the subjects were well controlled with Crohn's Disease Activity Index (CDAI) scores lower than 150. There were no differences found between the caries and periodontal status between the control and CD groups. To examine the oral and gut microbiome, 50 saliva and 50 fecal samples were processed, DNA extracted and sequenced using whole genome shotgun sequencing.

\section{Gut microbiome profile and differentially abundant species}

Many commensal gut microbial species were found present at high abundances (>5\%) in both CD and healthy controls. These include Bacteroides dorei (11.2\%), Prevotella copri (9.1\%), Faecalibacterium prausnitzii (8.0\%), and Bacteroides uniformis (5.8\%) (Fig. 1a). There was no difference noted in alpha diversity at species level.

The species that were found to be significantly depleted (relative abundance) in CD compared to control include: F. prausnitzii (Median [Range]: 0.03 [0.00-4.56] vs 13.69 [5.32-18.71], $\mathrm{p}=0.010)$, Roseburia inulinivorans (Median [Range]: 0.00 [0.00-0.03] vs 0.21 [0.01-0.53], $\mathrm{p}=0.010$ ) and Alistipes senegalensis (Median [Range]: 0.00 [0.00-0.00] vs 0.00 [0.00-0.02], $\mathrm{p}=0.029$ ), while Clostridium nexile (Median [Range]: 0.00 [0.00-0.12] vs 0.00 [0.00-0.00], $\mathrm{p}=0.038$ ) and Ruminococcus gnavus (Median [Range]: 0.43 [0.02-0.33] vs 0.00 [0.00-0.13], $\mathrm{p}=0.043$ ) were found to be significantly enriched in subjects with CD compared to control (Fig. 2) (Table 2). 
Table 1 Demographics, clinical and oral conditions of subjects $(n=50)$

\begin{tabular}{|c|c|c|}
\hline & Crohn's disease (CD) & Healthy controls ( $\mathrm{HC})$ \\
\hline Mean age* (Range) & $40(23-67)$ & $40(21-66)$ \\
\hline \multicolumn{3}{|l|}{ Race } \\
\hline Han Chinese & 13 & 13 \\
\hline Malay & 3 & 3 \\
\hline Indian & 9 & 9 \\
\hline \multicolumn{3}{|l|}{ Sex } \\
\hline Male & 10 & 10 \\
\hline Female & 15 & 15 \\
\hline \multicolumn{3}{|l|}{ Oral condition } \\
\hline Mean $\mathrm{DMFT}^{\wedge}$ & $6.5 \pm 7.0$ & $4.6 \pm 5.0$ \\
\hline Periodontitis present & 5 & 5 \\
\hline \multicolumn{3}{|l|}{ Smoking } \\
\hline Non-smoker & 20 & 21 \\
\hline Previous/current smoker & 5 & 4 \\
\hline \multicolumn{3}{|l|}{ CD condition } \\
\hline Length of diagnosis in years (range) & $7.8(1-20)$ & \\
\hline Crohn's Disease Activity Index (CDAI) & $86.2 \pm 87.1$ & \\
\hline $\begin{array}{l}\text { Current disease activity: Active (Diarrhoea, Abdominal pain, Blood-in- } \\
\text { stool, fever and fatigue) }\end{array}$ & 10 & \\
\hline \multicolumn{3}{|l|}{ Drug regimen ${ }^{\#}$} \\
\hline Steroids/Anti-inflammatory (Mesalazine) & 8 & \\
\hline Azathioprine & 13 & \\
\hline Biologics & 11 & \\
\hline Others (Tacrolimus, sulphasalazine) & 2 & \\
\hline
\end{tabular}

Principal coordinate analysis (Fig. 3a) revealed visuallyderived groups of healthy controls driven by Prevotella copri and F. prausnitzii, while a group of CD subjects was characterized by Escherichia coli, Streptococcus salivarius and Lachnospiraceae (Wilcoxon test, p-value $\leq 0.0025$ ).

\section{Oral microbiome profile and differentially abundant species}

The oral microbiome was less dominated by specific species as compared to the gut microbiome. Microbes present at high abundance include Rothia mucilaginosa (12.0\%), Haemophilus parainfluenzae (10.3\%), and Neisseria $s p$. (5.7\%). There was also no difference noted in alpha diversity at species level between CD and control (Fig. 1b).

Interestingly, several Streptoccocus sp. and Human Herpesvirus 4 were found to be enriched and Bacteroides sp found to be depleted in subjects with CD; however, they were not significantly different after multiple testing correction (Table 2).

Principal coordinate analysis conducted on the species found in the oral microbiome did not reveal distinct visual clusters based on CD status. The microbiome also did not cluster based on oral conditions such as caries or periodontal disease (Fig. 3b).

\section{Relationship between gut and oral microbiome}

Out of the 50 pairs of gut and oral samples, Streptoccous salivarius were detected in 19 libraries; 7 gut and 12 oral samples. Among these, S. salivarius was detected in both the gut and oral samples in 4 subjects, all with CD. A strain typing analysis based on multiple sequence alignment of marker genes of $S$. salivarius in the subjects showed that the gut and saliva strains from the same subject clustered in close distance despite the samples being sampled separately (Fig. 4 and Additional file 1: Table S1). This suggests the $S$. salivarius found in the saliva and gut were colonised by similar strains. This finding was not seen in the healthy controls.

Clinical characteristics of the subjects with concordant gut and oral S. salivarius strains were compared (Table 3). Subjects with higher CDAI scores $(<150)$ indicating active disease were significantly more likely to have concordant strains of S. salivarius $(p=0.012)$. 


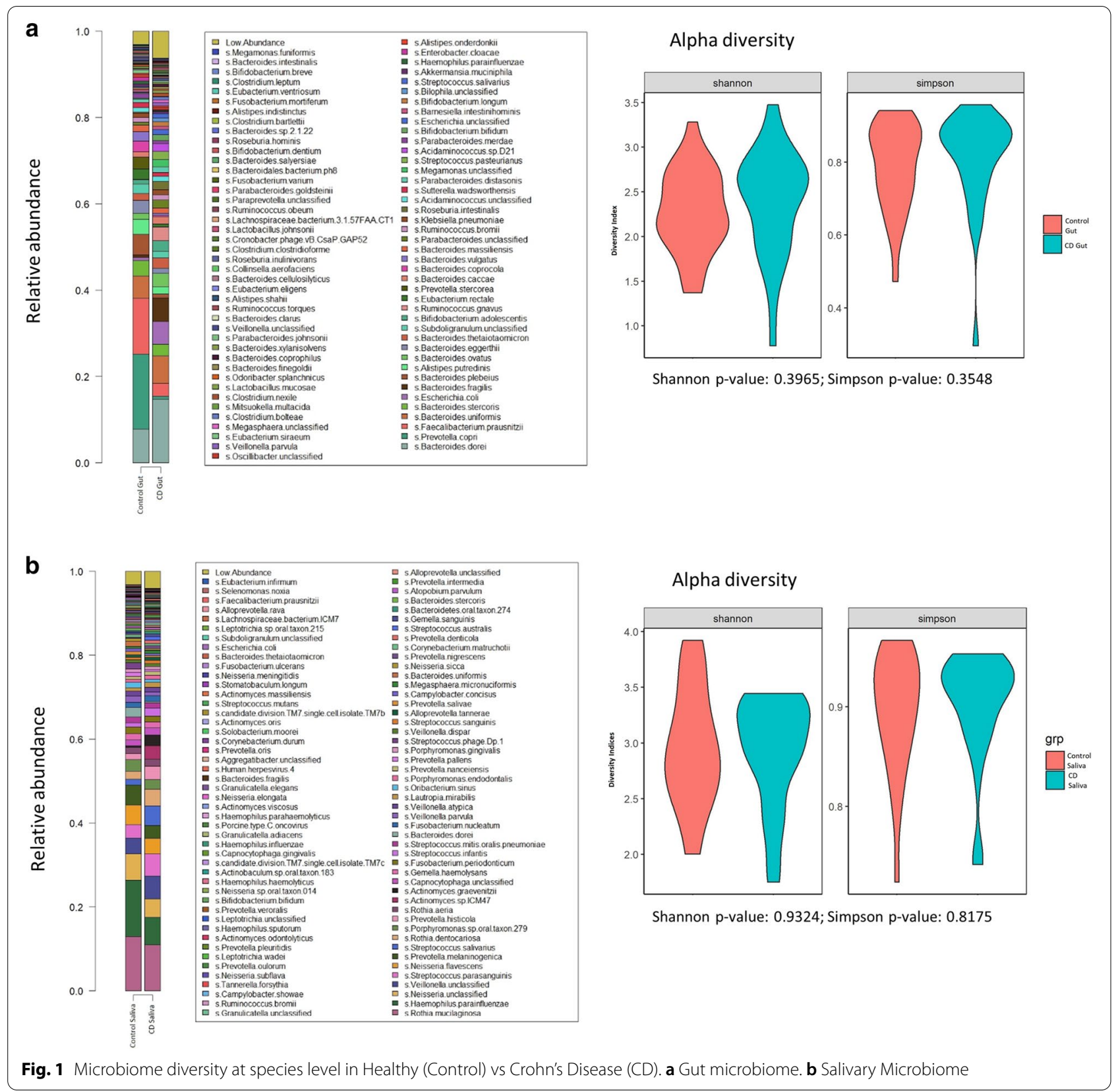

This was also reflected in subjects with signs and symptoms of active disease (Diarrhoea/abdominal pain/blood-in-stool/fever and fatigue) at the time of sampling $(p=0.016)$. Other clinical characteristics such as age, sex, race and oral conditions did not result in having more likely concordant gut and oral strains. Additionally, the use of proton pump inhibitors did not result in having more likely concordant gut and oral strains.

\section{Differentially abundant microbial pathways}

The HUMAnN2 analysis detected 395 pathways in the gut microbiome, of which 33 had Linear-discriminantanalysis (LDA) scores $\geq 3$ (Table 4). The top 4 pathways with higher relative abundance levels found in $\mathrm{CD}$ compared to control were related to the biosynthesis of arginine: L-arginine biosynthesis II (acetylcycle) $(\mathrm{LDA}=3.162, \mathrm{p}=0.001)$, L-ornithine biosynthesis $(\mathrm{LDA}=3.084, \mathrm{p}=0.001$ ), L-arginine biosynthesis I (via 


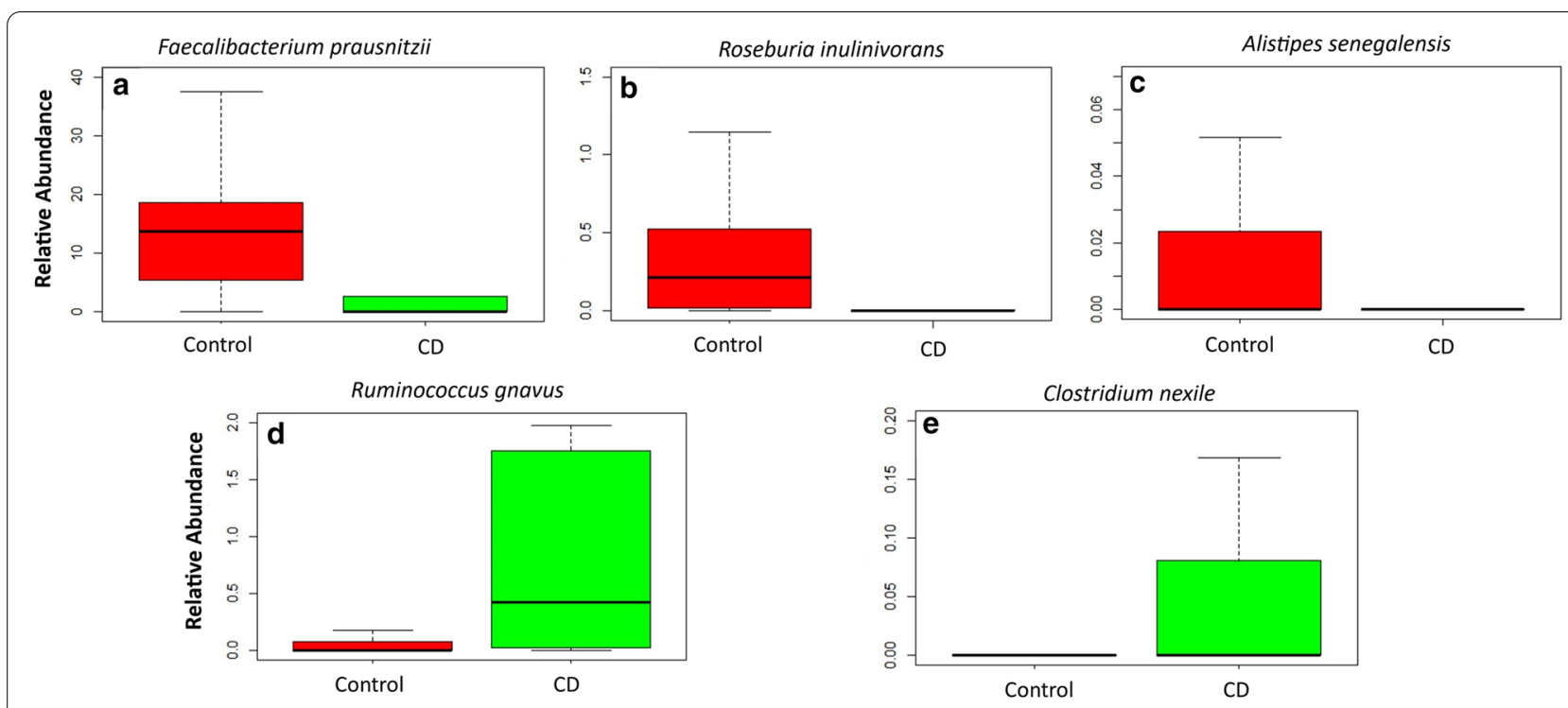

Fig. 2 Gut microbiome species relative abundance in Healthy (Control) vs Crohn's Disease (CD). a Faecalibacterium prausnitzii. b Roseburia inulinivorans. c Alistipes senegalensis. d Ruminococcus gnavus. e Clostridium nexile

L-ornithine) $(\mathrm{LDA}=3.077, \mathrm{p}=0.006)$ and $\mathrm{L}$-arginine biosynthesis IV (archaebacterial) (LDA $=3.059, \mathrm{p}=0.008$ ). The other higher relative abundance level pathways involve isoprene biosynthesis ( 2 pathways) (LDA $=3.058$ and 3.042, $\mathrm{p}=<0.001$ and $<0.001)$ and glycolysis (2 pathways) $(\mathrm{LDA}=3.038$ and $3.023, \mathrm{p}=<0.001$ and 0.001$)$. While the lower relative abundance level pathways found in $\mathrm{CD}$ compared to control include the S-adenosyl-L-methionine cycle I $(\mathrm{LDA}=3.550, \mathrm{p}<0.001)$, UMP biosynthesis (LDA $=3.434, \mathrm{p}<0.001)$ and 2 L-lysine biosynthesis pathways (LDA $=3.471$ and $3.421, \mathrm{p}=<0.001$ and 0.001).

In the oral microbiomes, 365 pathways were detected, of which 2 had LDA scores $\geq 3$ (Table 3). In CD, the superpathway of purine nucleotide salvage was found to have a higher relative abundance level while the superpathway of fatty acid biosynthesis initiation (E. coli) had a lower relative abundance level than the control group.

\section{Discussion}

This study represents a metagenomic insight into the oral and gut microbiome in CD patients, conducted in an Asian population, consisting subjects of Chinese, Indian and Malay descent (Table 1), which may have innate differences in gut and oral microbiome. Although some studies have examined the gut microbiome in CD [10, 21 , none of the previous studies on the oral microbiome employed the shotgun metagenomic technique used in this study. Furthermore, this is the first metagenomic study of matched oral and gut samples in CD subjects, allowing matching of the gut and oral microbiome at the strain level.

Crucially, this study found a cluster of CD subjects whose gut microbiome were characterized by $S$. salivarius, a prominent oral microbe. In health, Streptococcus genus typically constitute less than $4 \%$ of gut microbiome [13], thus it was note-worthy to find S. salivarius enriched in the gut microbiome of $C D$ subjects. Although the ectopic colonization of oral bacteria such as Klebsiella pneumoniae has been found to induce intestinal inflammation and can result in the progression of CD [14], it was only demonstrated in mouse models. Other studies suggesting roles in CD by oral bacteria were largely based on finding typically oral bacteria in the gut microbiome of $C D$ subjects. In the current study, matched oral and gut samples were taken and shown for the first time in CD subjects that $S$. salivarius were of similar strains. This increases the likelihood that the oral microbiome was the source for ectopic gut colonization in CD. This study also found that subjects in the active phase of disease were more likely to have ectopic gut colonization from the oral microbiome. A previous study found that oral microbes can colonize the gut after diarrheal episodes [15]. The authors hypothesized that the loss of gut microbes during diarrheal episodes reduces bacterial competition, this coupled with a transient increase in oxygen, allows for the ectopic colonization of oral microbes. Diarrhea is a major symptom during the active phase $C D$, suggesting that the oral microbiome can serve as a reservoir for pathogenic recolonization of the gut. A factor in ectopic gut colonization is the use of proton pump inhibitors 
Table 2 Species level differences between Crohn's disease and Control

\begin{tabular}{|c|c|c|c|c|c|}
\hline \multicolumn{3}{|l|}{ Depleted in crohn's disease } & \multicolumn{3}{|l|}{ Enriched in crohn's disease } \\
\hline Species & p-value & Adjusted p-value & Species & p-value & Adjusted p-value \\
\hline \multicolumn{6}{|l|}{ Gut } \\
\hline Faecalibacterium prausnitzii* & $<0.001$ & 0.010 & Clostridium nexile* & $<0.001$ & 0.038 \\
\hline Roseburia inulinivorans* & $<0.001$ & 0.010 & Ruminococcus gnavus* & 0.001 & 0.043 \\
\hline Alistipes senegalensis* & $<0.001$ & 0.029 & Bifidobacterium breve & 0.001 & 0.060 \\
\hline Bacteroides faecis & 0.001 & 0.060 & Escherichia unclassified & 0.003 & 0.105 \\
\hline Coprococcus catus & 0.002 & 0.083 & Escherichia coli & 0.004 & 0.115 \\
\hline Ruminococcus callidus & 0.002 & 0.099 & Anaerostipes unclassified & 0.005 & 0.115 \\
\hline Eubacterium rectale & 0.003 & 0.100 & Streptococcus anginosus & 0.005 & 0.115 \\
\hline Parabacteroides merdae & 0.005 & 0.115 & Streptococcus pasteurianus & 0.005 & 0.115 \\
\hline Bacteroides vulgatus & 0.006 & 0.125 & Coprobacillus unclassified & 0.009 & 0.149 \\
\hline Eubacterium eligens & 0.007 & 0.140 & Atopobium parvulum & 0.010 & 0.149 \\
\hline Dorea longicatena & 0.007 & 0.140 & Fusobacterium nucleatum & 0.010 & 0.149 \\
\hline Megamonas hypermegale & 0.010 & 0.149 & Blautia producta & 0.021 & 0.208 \\
\hline Alistipes putredinis & 0.010 & 0.149 & Erysipelotrichaceae bacterium & 0.021 & 0.208 \\
\hline Catenibacterium mitsuokai & 0.010 & 0.149 & Morganella morganii & 0.021 & 0.208 \\
\hline Bacteroides coprocola & 0.012 & 0.163 & Streptococcus macedonicus & 0.021 & 0.208 \\
\hline Paraprevotella unclassified & 0.013 & 0.163 & Proteus mirabilis & 0.021 & 0.208 \\
\hline Coprococcus comes & 0.013 & 0.163 & Veillonella atypica & 0.026 & 0.232 \\
\hline Peptostreptococcaceae unclassified & 0.014 & 0.170 & Acidaminococcus fermentans & 0.041 & 0.268 \\
\hline Bacteroides plebeius & 0.018 & 0.208 & Acidaminococcus sp D21 & 0.041 & 0.268 \\
\hline Eubacterium ventriosum & 0.021 & 0.208 & Clostridium innocuum & 0.041 & 0.268 \\
\hline Bacteroides stercoris & 0.023 & 0.219 & Lachnospiraceae bacterium & 0.041 & 0.268 \\
\hline Alistipes finegoldii & 0.024 & 0.222 & Lactobacillus gasseri & 0.041 & 0.268 \\
\hline Alistipes shahii & 0.025 & 0.228 & Lactobacillus mucosae & 0.041 & 0.268 \\
\hline Bacteroides nordii & 0.027 & 0.236 & Peptostreptococcus anaerobius & 0.041 & 0.268 \\
\hline Odoribacter splanchnicus & 0.028 & 0.236 & Prevotella histicola & 0.041 & 0.268 \\
\hline Eubacterium siraeum & 0.031 & 0.260 & Scardovia wiggsiae & 0.041 & 0.268 \\
\hline Holdemania unclassified & 0.037 & 0.268 & Streptococcus mutans & 0.041 & 0.268 \\
\hline Lachnospiraceae bacterium 5_1_63FAA & 0.038 & 0.268 & Rothia dentocariosa & 0.043 & 0.273 \\
\hline Barnesiella intestinihominis & 0.049 & 0.297 & Clostridium ramosum & 0.048 & 0.297 \\
\hline Roseburia hominis & 0.049 & 0.297 & & & \\
\hline \multicolumn{6}{|l|}{ Saliva } \\
\hline Bacteroides uniformis & $<0.001$ & 0.117 & Human herpesvirus 4 & 0.002 & 0.117 \\
\hline Bacteroides dorei & 0.001 & 0.117 & Streptococcus anginosus & 0.002 & 0.117 \\
\hline Haemophilus parainfluenzae & 0.003 & 0.128 & Streptococcus oligofermentans & 0.002 & 0.117 \\
\hline Subdoligranulum unclassified & 0.023 & 0.526 & Streptococcus vestibularis & 0.002 & 0.117 \\
\hline Prevotella salivae & 0.033 & 0.526 & Streptococcus cristatus & 0.004 & 0.134 \\
\hline \multirow[t]{12}{*}{ Prevotella intermedia } & 0.039 & 0.526 & Peptostreptococcus stomatis & 0.010 & 0.326 \\
\hline & & & Streptococcus salivarius & 0.020 & 0.526 \\
\hline & & & Actinomyces sp. ICM47 & 0.021 & 0.526 \\
\hline & & & Actinomyces naeslundii & 0.032 & 0.526 \\
\hline & & & Stomatobaculum longum & 0.033 & 0.526 \\
\hline & & & Actinomyces georgiae & 0.041 & 0.526 \\
\hline & & & Lactobacillus fermentum & 0.041 & 0.526 \\
\hline & & & Olsenella_uli & 0.041 & 0.526 \\
\hline & & & Oribacterium sp. Oral taxon 078 & 0.041 & 0.526 \\
\hline & & & Parvimonas micra & 0.041 & 0.526 \\
\hline & & & Slackia unclassified & 0.041 & 0.526 \\
\hline & & & Streptococcus peroris & 0.046 & 0.564 \\
\hline
\end{tabular}



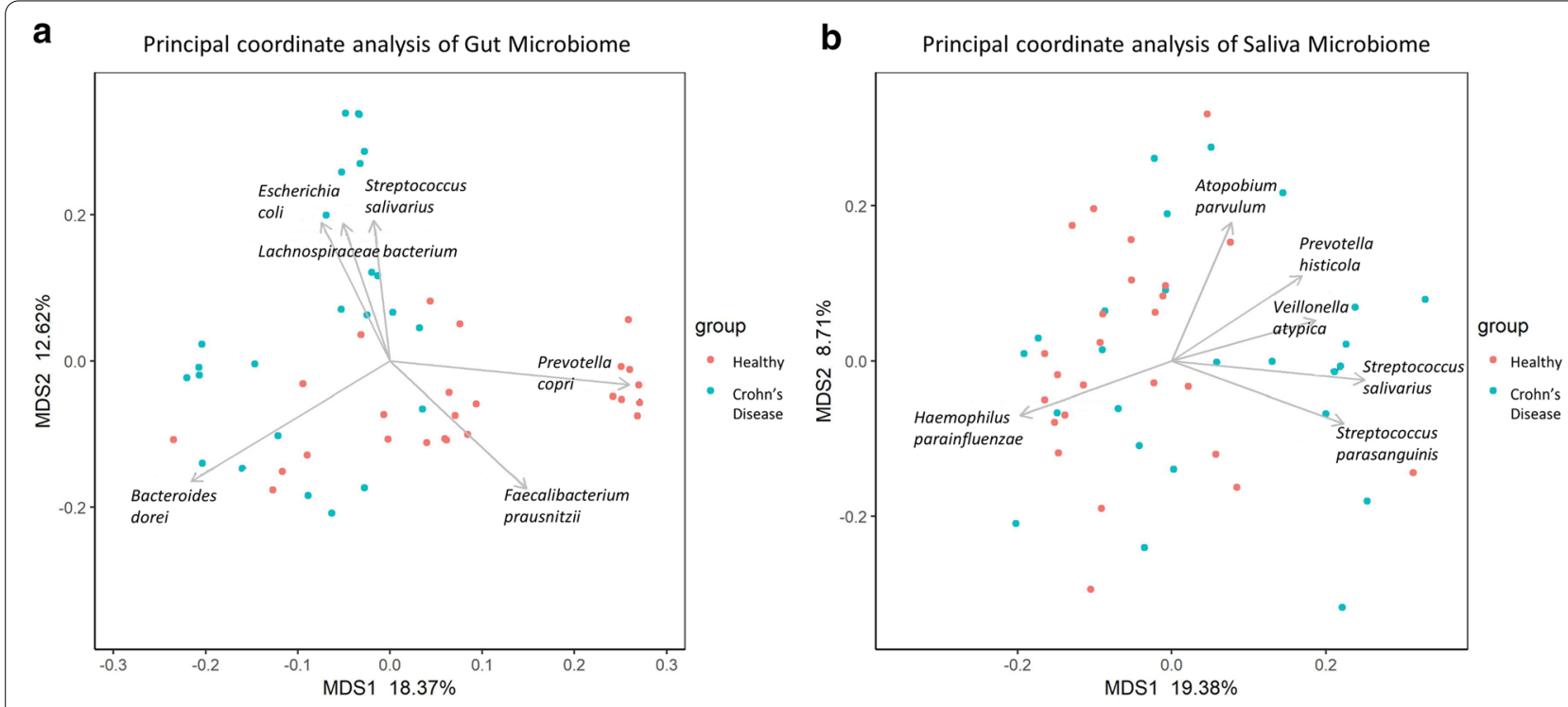

MDS: Multi-dimensional scaling based on Bray-Curtis distance

Fig. 3 Principal coordinate analysis of microbiome in Healthy (Control) vs Crohn's Disease (CD). a Gut microbiome. b Salivary Microbiome. The arrows drawn are for the top 6 most significant species at $p$-value $\leq 0.0025$

which decreases the gastric acidity, allowing for ectopic gut colonization of oral microbes [22]. However, this study did not find that the use of proton pump inhibitors and low gastric acid state increases the likelihood of ectopic gut colonization. A limitation of the study is the small number of subjects in which concordant gut and oral strains were found. Furthermore, although not statistically significant, all CD subjects with concordant gut and oral S. salivarius were Han Chinese. Therefore, it cannot be ruled out that racial differences [11] contributed to the finding of ectopic gut colonization. Larger follow up studies collecting both oral and gut samples will be required to expand upon this finding.

The gut microbial species found at high abundances $(>5 \%)$ in both $\mathrm{CD}$ and healthy controls in this study were similar to previous studies employing WGS [10, 23]. Contrary to previous studies, this study did not find significant reduction of alpha-diversity in the gut microbiome of CD subjects [24, 25]. However, most of these studies used 16S rRNA sequencing, which may be the reason for the difference. Additionally, another aspect of the study was that most of the CD subjects were in remission $(\mathrm{CDAI}<150)$ and well-controlled with medication, which may be a reason for their microbiome being less divergent from control. However, this would mean that any changes detected are more likely to be key changes driving microbiome dysbiosis.

The differentially abundant species found in gut microbiomes here were similar to previous studies. The depletion of butyrate-producing species such as $F$. prausnitzii and $R$. inulinivorans has been described in CD subjects [26]. F. prausnitzii in particular has been proposed to have protective effects in $C D[27,28]$, as the butyrate produced by these microbes aid in mucosal barrier function and maintenance of gut health [23]. Although Prevotella $s p$. has been studied extensively and some species have been shown to induce colitis in mice, it has yet been found to be associated with CD in humans [29]. In this study, it was found that $P$. copri was detected in a discreet cluster of healthy controls, similar to the previous WGS study [10]. This suggests that $P$. copri may be protective in some individuals against $\mathrm{CD}$ instead. A. senegalensis is a newly delineated species [30], which has yet to be implicated in CD. A related species, Alistipes putredinis has been described to contribute to transcriptional pathways in IBD [23]. Interestingly, it was also found to be significantly depleted in CD subjects in a previous WGS study [10] warranting further investigation. Additionally, a commonly implicated microbe Ruminococcus gnavus [23, 31] was found to be significantly enriched in CD subjects 


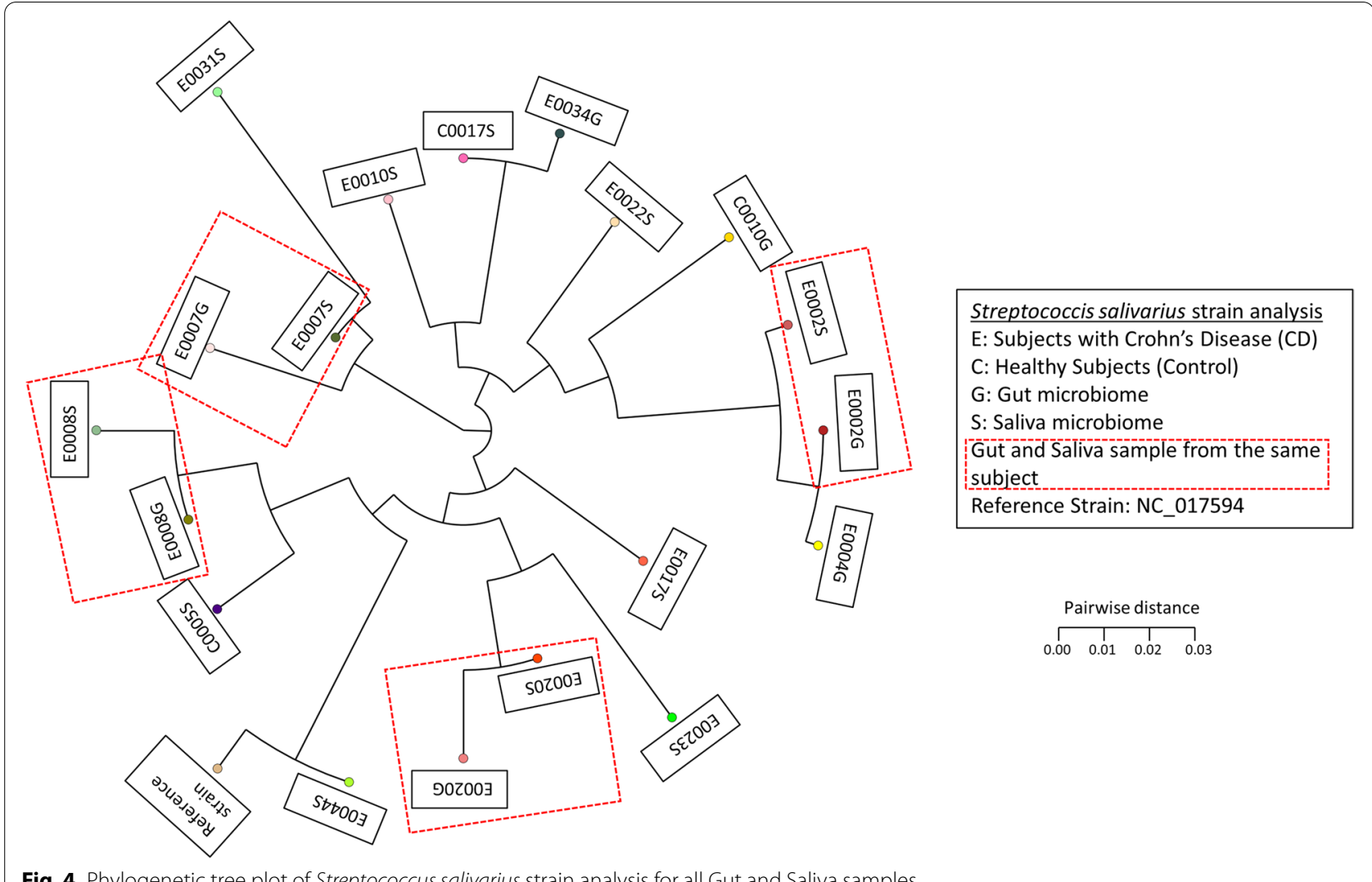

Fig. 4 Phylogenetic tree plot of Streptococcus salivarius strain analysis for all Gut and Saliva samples

in this study. A recent mechanistic study into R. gnavus showed that it produces a complex polysaccharide that can result in the pattern of inflammation seen in CD [32]. Another species implicated in the pathogenesis of $C D$ is $E$. coli, which was found to work in conjunction with other microbes as well as by exhibiting virulence features in $\mathrm{CD}$ subjects [31]. Although Clostridium nexile was found to be enriched in CD subjects in this study, it was found to be decreased in previous studies [33]. Comparing this to another WGS study, C. nexile was also found to be enriched in CD subjects, although not significantly [10]. C. nexile is able to produce short-chain fatty acids which decreases inflammation and alleviate colitis in experimental models [34]. However, the exact mechanism of that action is still unknown and does not explain why $C$. nexile was enriched instead in $\mathrm{CD}$. This suggests that $C$. nexile has a geographically or ethnically specific role and its function warrants further investigation.

Experts in the field have suggested of use the salivary microbiome as a non-invasive tool in the diagnosis of CD $[18,19]$. This study did not find any significant differences between the oral microbiome in $\mathrm{CD}$ and controls after adjustment, which could be due to the $\mathrm{CD}$ subjects being under treatment, with microbiome alterations due to medications rather than a signature of disease. However, when examining the genus differences, the study found similar enrichment of Streptococcus, and depletion of Haemophilus and Prevotella in CD subjects from previous $16 \mathrm{~S}$ studies $[18,19]$. Additionally, the ability to detect changes at species level provided better resolution than previous studies showing that the enrichment of Streptococcus genus involved several species such as $S$. anginosus, S. oligofermentans, S. vestibularis, S. cristatus and S. salivarius, while the depletion of Prevotella involved $P$. salivae and P. intermedia.

Another interesting finding from the salivary analysis is the enrichment of Human Herpesvirus 4, also known as Epstein-Barr virus (EBV), found in CD subjects. Recent studies have found a significant correlation between EBV presence in the gut with clinical disease severity [35]. This could be due to increased susceptibility of the CD patients on immunosuppressive treatment and the ability of EBV to induce inflammation. Being able to detect this in the oral microbiome may allow it to serve as a marker for disease severity.

The number of microbial pathways detected in this study was similar to a previous WGS study which 
Table 3 Clinical characteristics of subjects with concordant gut and oral S. salivarius strains

\begin{tabular}{|c|c|c|c|}
\hline & $\begin{array}{l}\text { CD subjects with concordant } \\
\text { strains }(n=4)\end{array}$ & $\begin{array}{l}\text { CD subjects with no concordant } \\
\text { strain }(n=21)\end{array}$ & $p$-value \\
\hline Crohn's Disease Activity Index (CDAl) $^{\#}$ & $184.3 \pm 2.9$ & $67.1 \pm 82.5$ & ${ }^{*} 0.012$ \\
\hline \multicolumn{4}{|l|}{ Disease status } \\
\hline $\begin{array}{l}\text { Active disease at time of sampling (Diarrhoea/abdominal } \\
\text { pain/blood-in-stool/fever and fatigue) }\end{array}$ & 4 & 6 & ${ }^{*} 0.016$ \\
\hline Inactive disease at time of sampling & 0 & 15 & \\
\hline Proton Pump Inhibitor use & & & 1.000 \\
\hline Yes & 1 & 7 & \\
\hline No & 3 & 14 & \\
\hline Mean age ${ }^{\#}$ & $38 \pm 14$ & $40 \pm 14$ & 0.774 \\
\hline Race & & & 0.056 \\
\hline Han Chinese & 4 & 9 & \\
\hline Malay & 0 & 3 & \\
\hline Indian & 0 & 9 & \\
\hline Sex & & & 0.142 \\
\hline Male & 3 & 7 & \\
\hline Female & 1 & 14 & \\
\hline Oral condition & & & 0.661 \\
\hline DMFT $^{\#}$ & $5.0 \pm 4.2$ & $6.8 \pm 7.4$ & \\
\hline Periodontitis & & & 0.451 \\
\hline Present & 1 & 4 & \\
\hline Absent & 3 & 17 & \\
\hline
\end{tabular}

All Fisher-Exact test except ${ }^{\#}$ T-test

*p-value $<0.05$

detected 255 pathways in CD subjects [10]. The microbial pathway analysis found that 4 pathways in the gut microbiome with higher relative abundance levels found in CD compared to control are related to arginine biosynthesis. The arginine pathway is a major contributor to host inflammatory processes via inducible nitric oxide $(\mathrm{NO})$ production, with $\mathrm{NO}$ providing protective cytostatic/cytotoxic antimicrobial action [36]. Administration of L-arginine has even been shown to reduce intestinal inflammation and pathology [37]. However, the specific role of microbial arginine metabolism has yet to be explored in the context of CD. It could be that these bacteria proliferate in the arginine poor environment in $\mathrm{CD}$ subjects as they are able to produce their own arginine. Additionally, isoprene biosynthesis was also found to be at higher relative abundance levels in CD subjects. The increase of isoprene in expired air from IBD subjects has been linked to the activity of disease, and is at a higher level than healthy individuals [38]. The microbial dysbiosis in CD favors a shift towards arginine and isoprene forming microbes providing a possible target to modify the microbiome.

\section{Conclusions}

The use of metagenomics has highlighted the relationship of the gut and oral microbiome in well-controlled CD subjects. The study found the evidence of ectopic gut colonization by oral bacteria in CD subject during active disease, suggesting that the oral microbiome could be a reservoir for pathogens in $\mathrm{CD}$ patients. In addition to corroborating previously implicated gut microbial differences such as $R$. gnavus and $F$. prausnitzii, this study also detected the depletion of the newly described $A$. senegalensis not previously implicated in $\mathrm{CD}$. Moreover, microbial arginine and isoprene pathways were found to be commonly present in CD gut microbiome, indicating that further study in this area is warranted.

\section{Methods}

\section{Subject inclusion and recruitment}

The study was conducted at the National University Hospital, Singapore from May 2017 to January 2019. Subjects with CD $(n=25)$ were recruited from the IBD clinic, Division of Gastroenterology \& Hepatology. Healthy 
Table 4 Microbial pathways with LDA effect $>3$

\begin{tabular}{|c|c|c|c|c|c|}
\hline \multicolumn{3}{|l|}{ Most common in control group } & \multicolumn{3}{|l|}{ Most common in crohn's disease group } \\
\hline Pathway & LDA effect & P-value & Pathway & LDA effect & P-value \\
\hline \multicolumn{6}{|l|}{ Gut } \\
\hline PWY 6151 S-adenosyl-L-methioninecycle I & 3.550 & $<0.001$ & $\begin{array}{l}\text { ARGSYNBSUB PWY L-arginine biosynthesis } \| \\
\text { (acetylcycle) }\end{array}$ & 3.162 & 0.001 \\
\hline PWY 5097 L-lysine biosynthesis VI & 3.471 & $<0.001$ & GLUTORN PWY L-ornithine biosynthesis & 3.084 & 0.001 \\
\hline PWY 5686 UMP biosynthesis & 3.434 & $<0.001$ & $\begin{array}{l}\text { ARGSYN PWY L-arginine biosynthesis I (via } \\
\text { L-ornithine) }\end{array}$ & 3.077 & 0.006 \\
\hline PWY 2942 L-lysine biosynthesis III & 3.421 & 0.001 & $\begin{array}{l}\text { PWY-7400 L-arginine biosynthesis IV (archaebacte- } \\
\text { rial) }\end{array}$ & 3.059 & 0.008 \\
\hline PWY 6700 queuosine biosynthesis & 3.414 & 0.002 & PWY 6270 isoprene biosynthesis I & 3.058 & $<0.001$ \\
\hline $\begin{array}{l}\text { NONMEVIPP PWY methylerythritol phosphate } \\
\text { pathway I }\end{array}$ & 3.414 & $<0.001$ & PWY 7560 methylerythritol phosphate pathway II & 3.042 & $<0.001$ \\
\hline PWY 6737 starch degradation V & 3.335 & 0.003 & GLYCOLYSIS glycolysis I from glucose-6-phosphate & 3.038 & $<0.001$ \\
\hline $\begin{array}{l}\text { PWY } 7219 \text { adenosine ribonucleotides de novo } \\
\text { biosynthesis }\end{array}$ & 3.296 & 0.001 & PWY66 400 glycolysis VI (metazoan) & 3.023 & 0.001 \\
\hline $\begin{array}{l}\text { PWY } 6386 \text { UDP N-acetylmuramoyl pentapeptide } \\
\text { biosynthesis II (lysine containing) }\end{array}$ & 3.234 & 0.003 & PWY 5484 glycolysis II from fructose-6-phosphate & 3.017 & 0.000 \\
\hline $\begin{array}{l}\text { PWY } 7221 \text { guanosine ribonucleotides de novo } \\
\text { biosynthesis }\end{array}$ & 3.219 & 0.004 & & & \\
\hline $\begin{array}{l}\text { PEPTIDOGLYCANSYN PWY peptidoglycan biosyn- } \\
\text { thesis I (meso-diaminopimelate containing) }\end{array}$ & 3.212 & 0.003 & & & \\
\hline $\begin{array}{l}\text { ILEUSYN PWY L-isoleucine biosynthesis I (from } \\
\text { threonine) }\end{array}$ & 3.212 & 0.006 & & & \\
\hline VALSYN PWY L-valine biosynthesis & 3.212 & 0.006 & & & \\
\hline $\begin{array}{l}\text { PWY } 6387 \text { UDP N-acetylmuramoyl pentapeptide } \\
\text { biosynthesis I (meso-diaminopimelate contain- } \\
\text { ing) }\end{array}$ & 3.170 & 0.003 & & & \\
\hline $\begin{array}{l}\text { BRANCHED CHAIN AA SYN PWY superpathway of } \\
\text { branched aminoacid biosynthesis }\end{array}$ & 3.132 & 0.008 & & & \\
\hline $\begin{array}{l}\text { GALACTUROCAT PWY D-galacturonate degrada- } \\
\text { tion I }\end{array}$ & 3.113 & $<0.001$ & & & \\
\hline PWY 6897 thiamin salvage II & 3.104 & 0.001 & & & \\
\hline $\begin{array}{l}\text { PWY } 7111 \text { pyruvate fermentation to isobutanol } \\
\text { engineered }\end{array}$ & 3.098 & 0.015 & & & \\
\hline PWY 5973 cis-vaccenate biosynthesis & 3.096 & $<0.001$ & & & \\
\hline $\begin{array}{l}\text { GALACT GLUCUROCAT PWY superpathway of } \\
\text { hexuronide and hexuronate degradation }\end{array}$ & 3.087 & 0.001 & & & \\
\hline $\begin{array}{l}\text { GLUCUROCAT PWY superpathway of beta } \\
\text { D-glucuronide and D-glucuronate degradation }\end{array}$ & 3.078 & 0.001 & & & \\
\hline PWY 5103 L-isoleucine biosynthesis III & 3.062 & 0.012 & & & \\
\hline $\begin{array}{l}\text { PWY 6507 4-deoxy-L-threo-hex-4-enopyranuro- } \\
\text { nate degradation }\end{array}$ & 3.040 & 0.001 & & & \\
\hline PWY 7242 D-fructuronate degradation & 3.019 & 0.003 & & & \\
\hline \multicolumn{6}{|l|}{ Saliva } \\
\hline $\begin{array}{l}\text { FASYN INITIAL PWY superpathway of fatty acid } \\
\text { biosynthesis initiation (E-coli) }\end{array}$ & 3.036 & 0.002 & $\begin{array}{l}\text { PWY66 } 409 \text { superpathway of purine nucleotide } \\
\text { salvage }\end{array}$ & 3.035 & 0.018 \\
\hline
\end{tabular}

controls $(n=25)$ without any signs and symptoms of $C D$ were age, sex and ethnicity matched and recruited from the Dental Centre, University Dental Cluster. The control subjects presented at the Dental Centre for routine dental care such as check-up, cleaning and minor restoration. Demographic information, history of tobacco use, other co-morbidities, current drug regimen and current disease activity were recorded. Current disease activity was calculated using the Crohn's Disease activity index (CDAI) as well as the presence of common signs and symptoms (Diarrhoea/abdominal pain/blood-in-stool/ fever and fatigue) [39]. Subjects who had antibiotic/ 
probiotic/prebiotic use within a month, actively treated for a malignancy with chemotherapy, or diagnosed with an indeterminate colitis were excluded from the study. Non-CD controls were further required to have no known gastrointestinal signs and symptoms such as diarrhoea, abdominal pain and blood-in-stool.

The study received ethics approval from the institutional review boards of the National Healthcare Group, Singapore (DSRB reference E/2016/01,285). Informed consent was obtained from all participants. Additionally, all experiments were performed in accordance with relevant guidelines and regulations.

\section{Oral condition of subjects}

Oral involvement of $\mathrm{CD}$ is well documented; with studies reporting between 20 and $50 \%$ of cases exhibiting oral manifestations including aphthous ulcers, linear deep ulcers, mucosal tags, mucosal "cobblestoning", mucogingivitis and lip swelling. The presence of these conditions can affect the oral microbiome [40]. In order to detect differences in the oral microbiome that are key drivers of $\mathrm{CD}$ progression, subjects exhibiting oral manifestations were excluded for this study. Other conditions that can affect the oral microbiome such as the presence of caries and periodontal disease were also recorded at sample collection. This was verified by an oral examination conducted by a dentist under artificial light.

\section{Sample collection}

Saliva was collected for the examination of the oral microbiome. Subjects were told to refrain from eating, smoking and dental procedures one hour prior to the collection. At least $5 \mathrm{ml}$ of resting whole saliva was collected using the OMNIgene Discover Kit 505 (DNA Genotek, Ottawa, ON, Canada) according to manufacturer's instructions.

Fecal sample was collected for the examination of the gut microbiome. Subjects were instructed and issued the OMNIgene•GUT (OMR-200) (DNA Genotek, Ottawa, ON, Canada) kit for sample collection. This was completed within two weeks of the saliva collection.

The oral and fecal samples were stored at room temperature as per manufacturer's recommendation and sent for DNA extraction within 4 weeks from collection.

\section{DNA extraction and purification}

Community DNA was extracted from saliva and fecal samples after mechanical lysis via bead-beating using Exgene $^{\mathrm{TM}}$ Clinic SV Mini kits (GeneAll Biotechnology, Dongnam-ro, Seoul, Korea), and QIAmp ${ }^{\circledR}$ FAST DNA Stool Mini kits (Qiagen, Hilden, Germany) respectively according to manufacturer's instructions. Extracted DNA was purified using AMPure XP beads (Beckman Coulter, Indianapolis, IN, USA). The quantity and quality of DNA was examined using a NanoDrop 8000 Spectrophotometer (Thermo Fisher Scientific, Carlsbad, CA, United States). Extracted DNA samples were stored at $-80{ }^{\circ} \mathrm{C}$ for up to 6 weeks prior to library preparation.

\section{Library preparation and Illumina sequencing}

Indexed sequencing libraries were prepared using QIAGEN $^{\circledR}$ QIAseq FX DNA Library Kit (Qiagen, Hilden, Germany) following manufacturer's instructions and sequenced as paired-end reads $2 \times 151 \mathrm{bp}$ on the Illumina HiSeq 4000 sequencer (Illumina, San Diego, CA, USA). On average, 15.5 million raw read pairs were obtained for each sample.

\section{Sequence data processing}

Sequenced reads for each library were de-multiplexed into individual fastq file, and analysed using a pipeline (https://github.com/gis-rpd/pipelines/tree/master/metag enomics/shotgun-metagenomics) for processing pairedend shotgun metagenomic sequencing data. Firstly, raw reads was analyzed using Skewer to trim-off adapter sequences and low-quality bases from each read [41]. After trimming, reads were decontaminated to remove genomic sequences from the human host by using BWAMEM to map reads against the hg19 reference [42]. The remaining reads were regarded as likely of microbial origin (average $=7$ million) and were used as input for subsequent taxonomic and functional profiling.

\section{Taxonomic profiling}

The MetaPhlAn2 software was used to profile the taxonomic composition of the microbial communities from the post quality-filtered reads [43]. Reads with sequences that matched microbial clades were used to normalize and calculate relative abundance for taxa from kingdom to species ranks. For reducing noise from false positive identifications, taxa with total relative abundance $<0.1 \%$ were excluded from further statistical analysis.

\section{Strain typing}

To examine the relationship of the oral microbiome to the gut microbiome, strain analysis on Streptoccous salivarius, a predominantly oral microbe, was conducted on all gut and oral samples using StrainPhlAn. Using the reads mapped against the marker database of MetaPhlAn2, we extracted the clade specific markers of $S$. salivarius. Subsequently multiple sequence alignment 
of the marker sequences of $S$. salivarius strains was performed among the gut and saliva libraries of samples as well as the reference genome (NC_017594), before building the phylogenetic tree [44].

\section{Statistical analysis Principal coordinates analysis (PCoA)}

Bray-Curtis distance was calculated for PCoA analysis based on the species profiled in the sample set, while the multi-dimensional scaling (MDS) dimensions 1 and 2 were used to visualize the level of similarity between samples based on their microbiome composition.

\section{Diversity analysis}

The Vegan package in $\mathrm{R}$ was used to calculate Shannon and Simpson's diversity values at the species level. Subsequently, ggplot2 was used to generate diversity plots. Wilcoxon test in $\mathrm{R}$ was used to test for significant difference in the medians of Shannon diversity values between case and control groups.

\section{Association analysis for taxonomic abundances with Crohn's disease}

The Wilcoxon test in $\mathrm{R}$ was used to compare the median relative abundance in the cases with Crohn's disease $(C D)$ versus the non-diseased control group for a significant difference. To analyze the direction of the association, the "alternative" function was used in the model.

\section{Multiple testing correction}

We adopted Benjamin Hochberg's false discovery rate method to correct for multiple testing at a significance threshold of $5 \%$.

\section{Pathway analysis}

The HMP Unified Metabolic Analysis Network (HUMAnN2) program was used to determine relative abundance of microbial pathways in gut and saliva microbiomes of $C D$ and control groups. The default Kyoto encyclopedia of genes and genomes (KEGG) orthology catalogue was used as the pathway reference. Subsequently, we extracted the total pathway abundance contributed by the genes present in every taxa of the community for analysis. For association, the Linear discriminant analysis Effect Size (LEfSe) software recognized the relative abundance of each pathway as the feature to test for significant difference in the medians between the two groups. We used the default LDA scores $\geq 2$ as the threshold for significance, and to highlight the significance of the association, a more stringent threshold at $\geq 3$ was also used for reporting [45].

\section{Supplementary Information}

The online version contains supplementary material available at https://doi. org/10.1186/s13099-021-00409-5.

Additional file 1: Table S1. Pairwise distance between S. salivarius strains gene markers analysis.

\section{Acknowledgements}

The authors would like to thank Dr Juanda Leo Hartono, Dr Chen Weihao, Ms Coka Yip, Mr Collins Wenhan Chu and Ms Ong Mei Teng for their valuable contributions in subject recruitment.

\section{Authors' contributions}

SH and EP are considered first authors. SH conceived the idea; $\mathrm{SH}, \mathrm{MG}$ and EO participated in collecting the samples; EP conducted the data analysis; SH, EP and NN led the writing; MG, EO, PFdS and JS revised the manuscript for important intellectual content. All authors read and approved the final manuscript.

\section{Funding}

This study is supported by a National University of Singapore Start-up grant (Exploring the Oral Microbiome in Crohn's Disease).

\section{Availability of data and materials}

Whole-genome sequencing data that support the findings of this study are available in the European Nucleotide Archive with the primary accession code PRJEB39813.

\section{Ethics approval and consent to participate}

The study received ethics approval from the institutional review boards of the National Healthcare Group, Singapore (DSRB reference E/2016/01285). Informed consent was obtained from all participants.

\section{Consent for publication}

Not applicable.

\section{Competing interests}

The authors declare that they have no competing interests.

\section{Author details}

${ }^{1}$ Discipline of Orthodontics and Paediatric Dentistry, Faculty of Dentistry, National University of Singapore, 9 Lower Kent Ridge Road, Singapore 119085, Singapore. ${ }^{2}$ Genome Institute of Singapore, Agency for Science, Technology and Research (A*STAR), 60 Biopolis St, Singapore 138672, Singapore. ${ }^{3}$ Division of Gastroenterology \& Hepatology, National University Hospital, 5 Lower Kent Ridge Rd, Singapore 119074, Singapore. ${ }^{4}$ Yong Loo Lin School of Medicine, National University of Singapore, Singapore, Singapore.

Received: 5 November 2020 Accepted: 18 February 2021

Published online: 25 February 2021

\section{References}

1. Kaplan GG. The global burden of IBD: from 2015 to 2025. Nat Rev Gastroenterol Hepatol. 2015;12(12):720-7.

2. Baumgart DC, Sandborn WJ. Crohn's disease. Lancet. 2012;380(9853):1590-605

3. Kappelman MD, Rifas-Shiman SL, Porter CQ, Ollendorf DA, Sandler RS, Galanko JA, et al. Direct health care costs of Crohn's disease and ulcerative colitis in US children and adults. Gastroenterology. 2008;135(6):1907-13.

4. Cheifetz AS. Management of active Crohn disease. JAMA. 2013;309(20):2150-8. 
5. Cosnes J, Nion-Larmurier I, Beaugerie L, Afchain P, Tiret E, Gendre JP. Impact of the increasing use of immunosuppressants in Crohn's disease on the need for intestinal surgery. Gut. 2005;54(2):237-41.

6. Hold GL, Smith M, Grange C, Watt ER, El-Omar EM, Mukhopadhya I. Role of the gut microbiota in inflammatory bowel disease pathogenesis: what have we learnt in the past 10 years? World J Gastroenterol. 2014;20(5):1192-210.

7. Bloom SM, Bijanki VN, Nava GM, Sun L, Malvin NP, Donermeyer DL, et al. Commensal Bacteroides species induce colitis in host-genotype-specific fashion in a mouse model of inflammatory bowel disease. Cell Host Microbe. 2011;9(5):390-403.

8. Lupp C, Robertson ML, Wickham ME, Sekirov I, Champion OL, Gaynor EC, et al. Host-mediated inflammation disrupts the intestinal microbiota and promotes the overgrowth of Enterobacteriaceae. Cell Host Microbe. 2007;2(2):119-29.

9. Frank DN, Robertson CE, Hamm CM, Kpadeh Z, Zhang T, Chen H, et al. Disease phenotype and genotype are associated with shifts in intestinalassociated microbiota in inflammatory bowel diseases. Inflamm Bowel Dis. 2011;17(1):179-84.

10. Lloyd-Price J, Arze C, Ananthakrishnan AN, Schirmer M, Avila-Pacheco J, Poon TW, et al. Multi-omics of the gut microbial ecosystem in inflammatory bowel diseases. Nature. 2019;569(7758):655-62.

11. Chen $L$, Zhang $Y H$, Huang T, Cai YD. Gene expression profiling gut microbiota in different races of humans. Sci Rep. 2016:6:23075.

12. Kitamoto S, Nagao-Kitamoto H, Jiao Y, Gillilland III MG, Hayashi A, Imai J, et al. The intermucosal connection between the mouth and gut in commensal pathobiont-driven colitis. Cell. 2020;182(2):447-62. e14

13. Kitamoto S, Nagao-Kitamoto H, Hein R, Schmidt TM, Kamada N. The bacterial connection between the oral cavity and the gut diseases. J Dent Res. 2020;99(9):1021-9.

14. Atarashi K, Suda W, Luo C, Kawaguchi T, Motoo I, Narushima S, et al. Ectopic colonization of oral bacteria in the intestine drives TH1 cell induction and inflammation. Science. 2017;358(6361):359-65.

15. The HC, Florez de Sessions P, Jie S, Pham Thanh D, Thompson CN, Nguyen Ngoc Minh C, et al. Assessing gut microbiota perturbations during the early phase of infectious diarrhea in Vietnamese children. Gut Microbes. 2018;9(1):38-54.

16. Docktor MJ, Paster BJ, Abramowicz S, Ingram J, Wang YE, Correll M, et al. Alterations in diversity of the oral microbiome in pediatric inflammatory bowel disease. Inflamm Bowel Dis. 2012;18(5):935-42.

17. Kelsen J, Bittinger K, Pauly-Hubbard H, Posivak L, Grunberg S, Baldassano $\mathrm{R}$, et al. Alterations of the Subgingival Microbiota in Pediatric Crohn's Disease Studied Longitudinally in Discovery and Validation Cohorts. Inflamm Bowel Dis. 2015;21(12):2797-805.

18. Said HS, Suda W, Nakagome S, Chinen H, Oshima K, Kim S, et al. Dysbiosis of salivary microbiota in inflammatory bowel disease and its association with oral immunological biomarkers. DNA Res. 2014;21(1):15-25.

19. Xun Z, Zhang Q, Xu T, Chen N, Chen F. Dysbiosis and ecotypes of the salivary microbiome associated with inflammatory bowel diseases and the assistance in diagnosis of diseases using oral bacterial profiles. Front Microbiol. 2018:9:1136.

20. Xu X, He J, Xue J, Wang Y, Li K, Zhang K, et al. Oral cavity contains distinct niches with dynamic microbial communities. Environ Microbiol. 2015;17(3):699-710.

21. He Q, Gao Y, Jie Z, Yu X, Laursen JM, Xiao L, et al. Two distinct metacommunities characterize the gut microbiota in Crohn's disease patients. Gigascience. 2017;6(7):1-11.

22. Schmidt TS, Hayward MR, Coelho LP, Li SS, Costea PI, Voigt AY, et al. Extensive transmission of microbes along the gastrointestinal tract. Elife. 2019;8.

23. Schirmer M, Franzosa EA, Lloyd-Price J, Mclver LJ, Schwager R, Poon TW, et al. Dynamics of metatranscription in the inflammatory bowel disease gut microbiome. Nat Microbiol. 2018;3(3):337-46.

24. Morgan XC, Tickle TL, Sokol H, Gevers D, Devaney KL, Ward DV, et al. Dysfunction of the intestinal microbiome in inflammatory bowel disease and treatment. Genome Biol. 2012;13(9):R79.
25. Wills ES, Jonkers DM, Savelkoul PH, Masclee AA, Pierik MJ, Penders J. Fecal microbial composition of ulcerative colitis and Crohn's disease patients in remission and subsequent exacerbation. PLoS ONE. 2014;9(3):e90981.

26. Takahashi K, Nishida A, Fujimoto T, Fujii M, Shioya M, Imaeda H, et al. Reduced abundance of butyrate-producing bacteria species in the fecal microbial community in crohn's disease. Digestion. 2016;93(1):59-65.

27. Sokol H, Pigneur B, Watterlot L, Lakhdari O, Bermudez-Humaran LG, Gratadoux JJ, et al. Faecalibacterium prausnitzii is an anti-inflammatory commensal bacterium identified by gut microbiota analysis of Crohn disease patients. Proc Natl Acad Sci U S A. 2008;105(43):16731-6.

28. Sokol H, Seksik P, Furet JP, Firmesse O, Nion-Larmurier I, Beaugerie L, et al. Low counts of Faecalibacterium prausnitzii in colitis microbiota. Inflamm Bowel Dis. 2009;15(8):1183-9.

29. Larsen JM. The immune response to Prevotella bacteria in chronic inflammatory disease. Immunology. 2017;151(4):363-74.

30. Mishra AK, Gimenez G, Lagier JC, Robert C, Raoult D, Fournier PE. Genome sequence and description of Alistipes senegalensis sp. nov. Stand Genomic Sci. 2012;6(3):1-16.

31. Hoarau G, Mukherjee PK, Gower-Rousseau C, Hager C, Chandra J, Retuerto MA, et al. Bacteriome and Mycobiome Interactions Underscore Microbial Dysbiosis in Familial Crohn's Disease. MBio. 2016;7(5).

32. Henke MT, Kenny DJ, Cassilly CD, Vlamakis H, Xavier RJ, Clardy J. Ruminococcus gnavus, a member of the human gut microbiome associated with Crohn's disease, produces an inflammatory polysaccharide. Proc Natl Acad Sci U S A. 2019;116(26):12672-7.

33. Gevers D, Kugathasan S, Denson LA, Vazquez-Baeza Y, Van Treuren W, Ren $B$, et al. The treatment-naive microbiome in new-onset Crohn's disease. Cell Host Microbe. 2014;15(3):382-92.

34. Zhuang X, Li T, Li M, Huang S, Qiu Y, Feng R, et al. Systematic review and meta-analysis: short-chain fatty acid characterization in patients with inflammatory bowel disease. Inflamm Bowel Dis. 2019;25(11):1751-63.

35. Nissen LH, Nagtegaal ID, de Jong DJ, Kievit W, Derikx LA, Groenen PJ, et al. Epstein-Barr virus in inflammatory bowel disease: the spectrum of intestinal lymphoproliferative disorders. J Crohns Colitis. 2015;9(5):398-403.

36. Satriano J. Arginine pathways and the inflammatory response: interregulation of nitric oxide and polyamines: review article. Amino Acids. 2004;26(4):321-9.

37. Fritz JH. Arginine cools the inflamed gut. Infect Immun. 2013;81(10):3500-2.

38. Kurada S, Alkhouri N, Fiocchi C, Dweik R, Rieder F. Review article: breath analysis in inflammatory bowel diseases. Aliment Pharmacol Ther. 2015;41(4):329-41.

39. Best WR, Becktel JM, Singleton JW, Kern F Jr. Development of a Crohn's disease activity index. National Cooperative Crohn's Disease Study Gastroenterology. 1976;70(3):439-44.

40. O'Brien CL, Kiely CJ, Pavli P. The microbiome of Crohn's disease aphthous ulcers. Gut Pathog. 2018;10:44.

41. Jiang H, Lei R, Ding SW, Zhu S. Skewer: a fast and accurate adapter trimmer for next-generation sequencing paired-end reads. BMC Bioinformatics. 2014;15:182.

42. Li H. Aligning sequence reads, clone sequences and assembly contigs with BWA-MEM. arXiv preprint. arXiv:1303.3997. 2013

43. Truong DT, Franzosa EA, Tickle TL, Scholz M, Weingart G, Pasolli E, et al. MetaPhIAn2 for enhanced metagenomic taxonomic profiling. Nat Methods. 2015;12(10):902-3.

44. Truong DT, Tett A, Pasolli E, Huttenhower C, Segata N. Microbial strainlevel population structure and genetic diversity from metagenomes. Genome Res. 2017;27(4):626-38

45. Abubucker S, Segata N, Goll J, Schubert AM, Izard J, Cantarel BL, et al. Metabolic reconstruction for metagenomic data and its application to the human microbiome. PLoS Comput Biol. 2012;8(6):e1002358.

\section{Publisher's Note}

Springer Nature remains neutral with regard to jurisdictional claims in published maps and institutional affiliations. 